\title{
MODELLING THE DYNAMIC RESPONSE OF CONCRETE WITH THE DAMAGE PLASTICITY MODEL CDPM2
}

\author{
PETER GRASSL \\ *University of Glasgow \\ Glasgow, UK \\ e-mail: peter.grass1@glasgow.ac.uk
}

Key words: Fracture, Concrete, Dynamic loading, Reinforcement, Damage-Plasticity

\begin{abstract}
Combinations of damage mechanics and the theory of plasticity have shown to be suitable for modelling concrete's quasi-brittle response in tension and low-confined compression, as well as the ductile response in confined compression. In this work, the recently proposed damage-plasticity constitutive model CDPM2 has been extended to the modelling of plain and reinforced concrete subjected to dynamic loading by making the damage part dependent on the strain rate. The approach is then applied to a dynamic compact tension test and a slab subjected to air blast loading for both of which experimental results have been reported in the literature.
\end{abstract}

\section{INTRODUCTION}

Concrete structures subjected to dynamic loading exhibit a complex nonlinear failure response which differs significantly from the one due to quasi-static loading. For instance, structures subjected to impact and blast can exhibit localised shear failure for loads, which, if they were applied slowly, would result in bending failure. Furthermore, dynamic loading produces compressive shock waves which can cause tensile spalling of the concrete cover, once they are reflected at free boundaries.

For improving design approaches for concrete structures subjected to dynamic loading, numerical techniques are useful since they allow for elucidating processes, which would not be easily measured by experiments. However, experiments are required to check that numerical techniques can reproduce important physical processes correctly.

Here, numerical techniques consisting of the explicit finite element method with an extended version of the damage-plasticity model CDPM2 reported in Grassl et al. (2011) and Grassl et al. (2013) are investigated. CDPM2 has been shown to provide good agreement with experimental results of concrete subjected to quasi-static loading in Grassl et al. (2013) and Xenos and Grassl (2016). However, the part of CDPM2 for modelling the dynamic response of concrete, which was originally proposed in Grassl et al. (2011), requires enhancements which are presented here.

For modelling the dynamic response of structures, an extension of CDPM2 is proposed. The performance of new model is then assessed by modelling a plain concrete compact tension test in Ožbolt et al. (2013) and a reinforced concrete slab subjected to air blast reported in Thiagarajan and Johnson (2014). The compact tension experiment, in which the influence of applied displacement rates on crack patterns and load capacity was studied, has been analysed before using 3D microplane models in Ožbolt et al. (2013) and plain strain damage models in Pereira et al. (2017). The reinforced concrete slab was part of a blind simulation competition, which was discussed in Schwer (2014b). 
It was also analysed in Ekström (2016) with the old version of CDPM2 proposed in Grassl et al. (2011). This reinforced concrete slab exhibits a complex response, which is strongly influenced by the properties of the reinforcement.

Each test on its own might not be sufficient to assess the performance of a concrete constitutive model. For instance, computationally very demanding models might be capable of reproducing well experimental results of small scale plain concrete specimens, but not be applicable to three-dimensional analyses of structural concrete components. On the other hand, the overall response of structural components might not provide enough information to discriminate the performance of the concrete constitutive model. In this paper, it is aimed to analyse both experiments with the same calibration strategy to assess if the new version of CDPM2 provides satisfactory results for both of these experiments.

\section{MODEL}

For the nonlinear finite element approach, an explicit solution method is applied. Concrete is modelled using constant strain tetrahedra with the proposed extension of the damage-plasticity model CDPM2 (Grassl et al. 2011, Grassl et al. 2013). For the reinforced concrete slab, steel reinforcement is modelled using frame elements with the modified Johnson-Cook plasticity material model (Johnson and Cook 1985). For the interaction between steel and concrete, a bondslip law based on Model Code 2010 (CEB-FIP 2012 ) is used. The models are embedded in the finite element program LS-DYNA. For steel and bond, the models are readily available in the software. For concrete, the extension of CDPM2 was incorporated by means of a usersubroutine. In the following sections, the main features of the constitutive model CDPM2, and the steel and bond model are discussed.

\subsection{CONCRETE MODEL}

In CDPM2, the stress evaluation is based on the damage mechanics concept of nominal and effective stresses. The nominal stress is evaluated by a combination of damage mechanics and elasto-plasticity. The effective stress is the stress in the undamaged material, which is determined from the elasto-plasticity part alone. For the nominal stress evaluation, tensile and compressive damage variables are applied to positive and negative components of the principal effective stress, respectively.

The plasticity part of the model is formulated in the effective stress space by means of HaighWestergaard stress coordinates, which are the volumetric effective stress $\bar{\sigma}_{\mathrm{v}}$, the length of the deviatoric effective stress $\bar{\rho}$ and the Lode angle $\bar{\theta}$ (Jirásek and Bažant 2002). The yield surface is based on an extension of the static strength envelope in Menétrey and Willam (1995), which is known to reproduce well experimental results of concrete subjected to multiaxial stress states. This static strength envelope is characterised by curved meridians and deviatoric sections varying from almost triangular in tension to circular in highly confined compression. A hardening function $q_{\mathrm{h}}$ is used to model the evolution of the yield surface in the pre- and post-peak regimes as shown in Figure 11. Here, these regimes are defined by the static strength envelope, which forms the peak. In the pre-peak regime, the yield surface is capped both in hydrostatic tension and compression. At peak, the static strength envelope proposed in Menétrey and Willam (1995) is reached, which is open in hydrostatic compression. In the post-peak regime, the yield surface further extends with the shape being similar to the strength envelope.

The hardening function $q_{\mathrm{h}}$ in the post-peak regime is controlled by the hardening modulus $H_{\mathrm{p}}$. The greater the value of $H_{\mathrm{p}}$, the smaller is the contribution of plasticity in the post-peak regime. In quasi-static simulations, damage is initiated once the strength envelope is reached. The damage initiation at a point is a function of maximum equivalent strains in tension and compression reached in the history. For the dynamic simulations in the present study, in which the strain rate dependence of strength is modelled, damage initiation is made dependent on the strain rate. The greater the strain rate is, the 


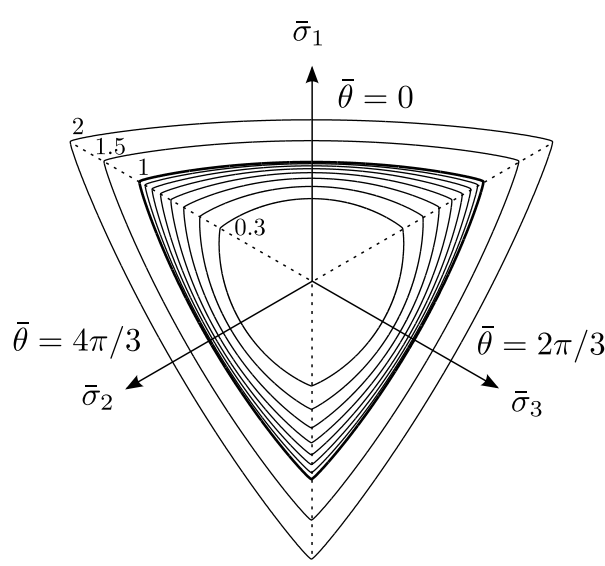

(a)

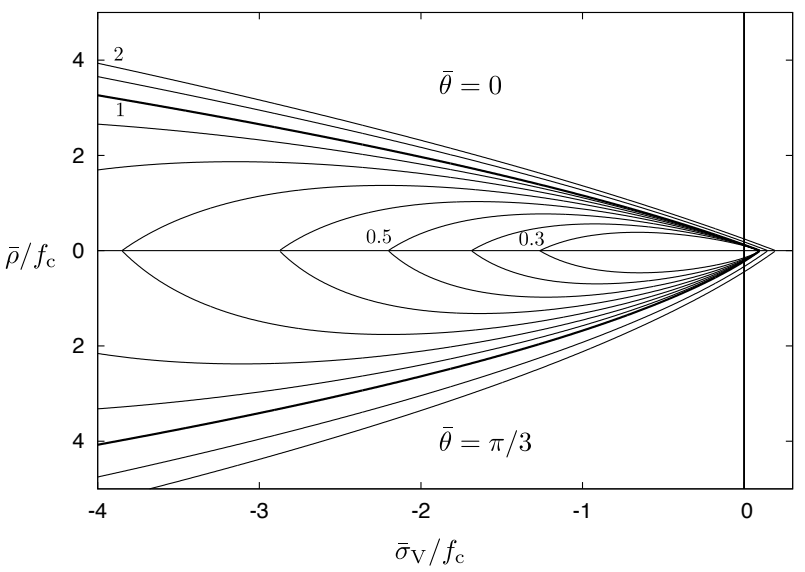

(b)

Figure 1: Evolution of the yield surface for varying values of the hardening variable $q_{\mathrm{h}}$ (from 0.3 to 2 ) which is less than one in the pre-peak and greater than one in the post-peak regime: (a) Deviatoric section for a constant volumetric stress of $\bar{\sigma}_{\mathrm{v}}=-f_{\mathrm{c}} / 3$ (b) meridians at $\theta=\pi / 3$ (compression) and $\theta=0$ (tension). Pre- and post-peak regimes are defined by the static strength surface $\left(q_{\mathrm{h}}=1\right)$, which is shown by a thicker line.

more plastic strains are generated before damage is initiated. This is achieved by dividing the rates of the equivalent strains by a strain rate dependent factor, which is equal to one for quasi-static loading and increases with increasing strain rate. Since the plastic part exhibits hardening (controlled by $H_{\mathrm{p}}$ ), the strength at which damage (and softening) is initiated exceeds the static strength of the envelope. This means that for dynamic loading, the pre- and post-peak regimes differ from those defined by the static strength envelope. For this technique to produce reasonable results in compression, it is required that the hardening modulus $H_{\mathrm{p}}$ is set to a large enough value so that the plastic strain before the onset of damage remains small. For dynamic simulations in which strain rate dependence of strength is considered, the recommended value of the hardening modulus is $H_{\mathrm{p}}=0.5$.

The strain rate dependent factors for tension and compression versus the strain rate are shown in Figure $2 \mathrm{a}$. They are based on the expressions in Model Code 10 (CEB-FIP 2012). However, only the initial branch of the expressions are used, since the steep branch for high strain rates is contributed to damage induced inertia effects as discussed in Cusatis (2011) and Ožbolt et al. (2014). It is assumed that these effects can be reproduced in finite element simulations automatically. This part of the model differs from the original proposal in Grassl et al. (2011), in which also the steep part was included.

Once damage is initiated, the response is a combination of the theory of plasticity and damage mechanics. Evolution laws for tensile and compressive damage are formulated as functions of positive and negative parts of the principal effective stress so that tensile and compressive softening responses can be described independently of each other. The function for the tensile damage variable is derived from a bilinear stress-crack opening $\left(\sigma-w_{\mathrm{c}}\right)$ curve, so that the results of analyses of tensile failure in which strains localise in mesh-dependent regions are independent of the finite element mesh (Pietruszczak and Mróz 1981, Bažant and Oh 1983, Willam et al. 1986). This bilinear curve is defined by the tensile strength $f_{\mathrm{t}}$ at zero crack opening, the stress threshold $f_{\mathrm{t} 1}$ at the crack opening threshold $w_{\mathrm{f} 1}$ and the crack opening threshold $w_{\mathrm{f}}$ at zero stress. In the crack band approach, the finer the mesh is, the larger the strain at constant damage is. This mesh dependent strain would result in a strong dependence of the mesh size on the strain rate effect on strength and fracture energy. Therefore, 


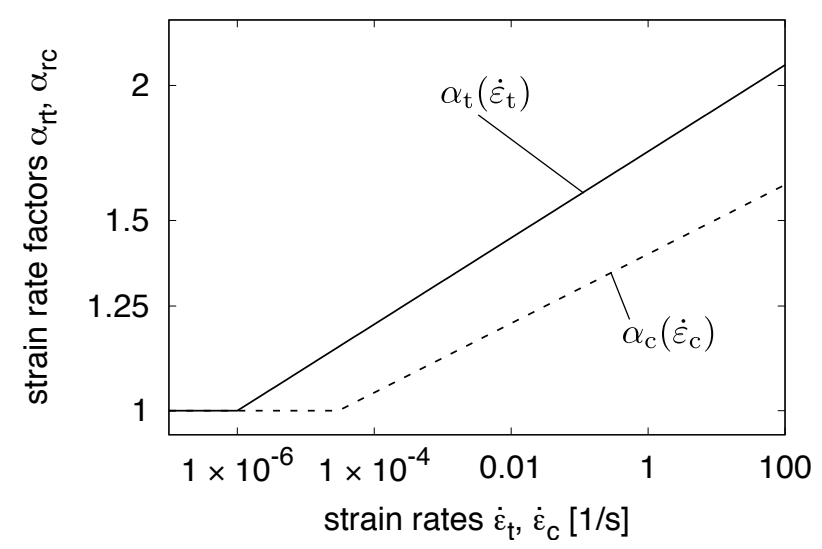

(a)

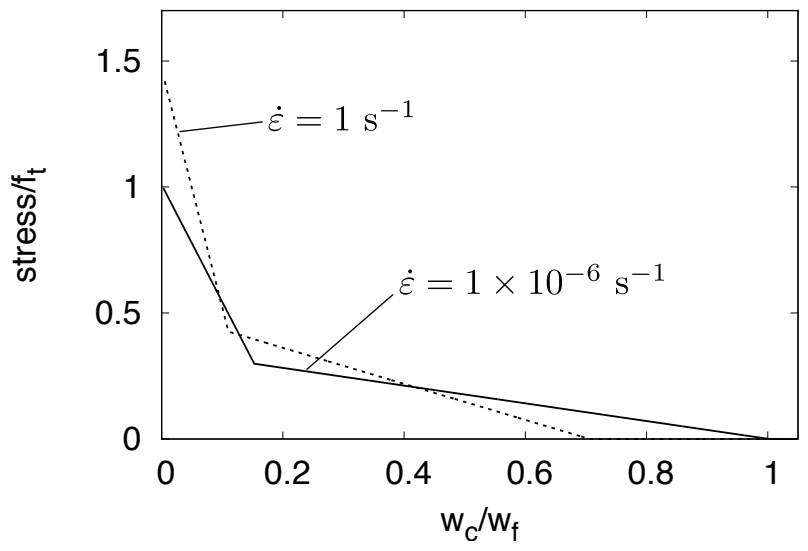

(b)

Figure 2: Strain rate dependence in CDPM2: (a) strain rate factor in uniaxial tension and compression modified from CEB-FIP (2012), (b) Bilinear stress-crack opening curve for quasi-static loading and moderate strain rate.

once damage is initiated, the strain rate dependent factor which is used to determine strain rate effect is set constant.

The tensile fracture energy of concrete $G_{\mathrm{F}}$ is defined as the area under the stress-crack opening curve. Not many experimental studies on the effect of strain rate on fracture energy are available (de Pedraza et al. 2018). Therefore, it is decided to keep the fracture energy independent of the strain rate. Within CDPM2, this is achieved by dividing the crack opening thresholds $w_{\mathrm{f}}$ and $w_{\mathrm{f} 1}$ by the square of the rate factor, so that the area below the stress-crack opening curve remains constant (Figure 2b). This approach differs from the original formulation in Grassl et al. (2011), where the crack threshold was not modified so that the model predicted a strong dependence of fracture energy on strain rate, which is not supported by experimental results.

For the present bilinear curve, the fracture energy is the area under stress-crack opening curve which is $G_{\mathrm{F}}=\left(f_{\mathrm{t}} w_{\mathrm{f} 1}+f_{\mathrm{t} 1} w_{\mathrm{f}}\right) / 2$. For the default in CDPM2, $f_{\mathrm{t} 1}=0.3 f_{\mathrm{t}}$ and $w_{\mathrm{f} 1}=$ $0.15 w_{\mathrm{f}}$, so that $G_{\mathrm{F}}=0.225 f_{\mathrm{t}} w_{\mathrm{f}}$. Thus, the crack opening threshold is related to fracture energy as $w_{\mathrm{f}}=4.444 G_{\mathrm{F}} / f_{\mathrm{t}}$. In the present finite element analyses, a crack band approach is applied for which the inelastic strain is determined as $\varepsilon_{\mathrm{c}}=w_{\mathrm{c}} / h_{\mathrm{e}}$, where $h_{\mathrm{e}}$ is a measure of the element length determined as a function of the volume of the element as $h_{\mathrm{e}}=\beta \sqrt[3]{V_{\mathrm{e}}}$. For direct tension tests using tetrahedral elements, factor $\beta$ must be chosen greater than one, otherwise the fracture energy obtained in simulations will be overestimated (Jirásek and Bauer 2012). In Grassl (2016), it was shown that for tetrahedral meshes used in a three-point bending analysis, $\beta=1.79$ provides a good representation of fracture energy, which is used in this study. The compressive damage variable is linked to a stress-inelastic strain curve, instead of a stress-crack opening curve, since the deformation patterns in the compressive zones of bending dominated applications are often meshsize independent (Grassl et al. 2013).

CDPM2 requires many input parameters, which can be divided into groups related to the elastic, plastic and damage parts of the model. In the present work, most of these parameters are set to their default values provided in Grassl et al. (2013), where it was shown that they provide a good match with experimental results. Some of the parameters which are directly linked to experimental results, such as density $\rho$, Young's modulus $E$, Poisson's ratio $\nu$, tensile strength $f_{\mathrm{t}}$, compressive strength $f_{\mathrm{c}}$, fracture energy $G_{\mathrm{F}}$ were adjusted to match material data available for the different groups of analyses. Furthermore, the default value for the inelastic strain threshold $\varepsilon_{\mathrm{cf}}$ results in a very brittle response in compression. Therefore, it 
is sometimes required to choose a more ductile compressive response to avoid premature failure in regions close to supports or applied loads by choosing a greater value for $\varepsilon_{\mathrm{cf}}$ than the default. This was done in the present study for the two tests.

\subsection{STEEL CONSTITUTIVE MODEL}

For the reinforcement steel, the modified Johnson-Cook constitutive model is used. The linear elastic part of the model requires as input parameters the Young's modulus $E$ and Poisson's ratio $\nu$. In the plastic part, the equation of the rate dependent yield strength is

$$
\sigma_{\mathrm{y}}=\left(A+B\left(\varepsilon_{\mathrm{eff}}^{p}\right)^{N}\right)(1+C \ln \dot{\varepsilon})
$$

where parameters $A, B$ and $N$ determine the hardening response and $C$ is used to model the strain rate dependence.

\subsection{BOND CONSTITUTIVE MODEL}

The bond-slip law describes the evolution of the bond stress with slip so that the force $F$ transferred by bond is

$$
F=\tau_{\mathrm{b}}(s) \pi \phi h_{\mathrm{m}}
$$

Here, the displacement $s$ is the slip, i.e. relative displacement, between the reinforcement and the concrete, $\phi$ is the diameter of the reinforcement and $h_{\mathrm{m}}$ is the average length of the reinforcement bar over which bond is acting. The function $\tau_{\mathrm{b}}(s)$ describes the bond-slip law, which is chosen as

$$
\tau_{\mathrm{b}}= \begin{cases}\tau_{\max }\left(\frac{s}{s_{\max }}\right)^{0.4} & \text { if } s<s_{\max } \\ \tau_{\max } & \text { if } s \geq s_{\max }\end{cases}
$$

where $\tau_{\max }$ is the bond strength and $s_{\max }$ is the slip at which the bond strength is reached. The first part of (3) for $s<s_{\max }$ is identical to the one in Model Code 2010 (CEB-FIP 2012) for ribbed bars with good bond conditions. The second part was chosen here to be constant, as done previously in Grassl et al. (2018). According to the Model Code 2010, the bond strength is $\tau_{\max }=2 \sqrt{f_{\text {ck }}}$. Here, the characteristic compressive strength $f_{\text {ck }}$ is obtained by subtracting $8 \mathrm{MPa}$ from the mean compressive strength.

\section{ANALYSES AND RESULTS}

Two concrete tests subjected to dynamic loading are analysed. In the first set of analyses, a compact tension specimen subjected to a range of prescribed displacement rates reported in Ožbolt et al. (2013) is studied. The experiments in Ožbolt et al. (2013) showed that the crack patterns in this compact tension test depended strongly on the displacement rate. The second test is a reinforced concrete slab subjected to air blast. The experimental results for this test were reported in Thiagarajan and Johnson (2014). In the following sections, the analyses of these tests are described.

\subsection{COMPACT TENSION TEST}

The first set of analyses is for a plain concrete compact tension test for which the experimental results were reported in Ožbolt et al. (2013). The geometry and loading setup of the test is shown in Figure 3 .

The setup consists of a notched concrete specimen loaded by two steel brackets connected to steel bars. The end of one of the bars is supported. To the other end a constant displacement rate is applied. In total, four displacement rates of $v=0.01,0.5,1.4$ and $4.3 \mathrm{~m} / \mathrm{s}$ are simulated in separate analyses. Both concrete specimen and loading brackets are meshed using tetrahedral elements of three different edge length of $h_{\mathrm{e}}=20,10$ and $5 \mathrm{~mm}$ to check that the model is able to reproduce the experimental results independent of the mesh size.

The input parameters for this test are determined using Model Code 2010 (CEB-FIP 2012) based on the uniaxial compressive strength $f_{\mathrm{c}}=$ $53 \mathrm{MPa}$ reported in Ožbolt et al. (2013). The resulting input parameters are $E=37 \mathrm{GPa}$, $f_{\mathrm{t}}=3.8 \mathrm{MPa}, G_{\mathrm{f}}=149 \mathrm{~J} / \mathrm{m}^{2}$. Poisson's ratio is set to $\nu=0.2$. Furthermore, the strain threshold for compressive softening is set to $\varepsilon_{\mathrm{fc}}=1 \times 10^{-3}$ to avoid premature crushing at the load application point. The other parameters are set to their default values for the strain rate dependent model with $H_{\mathrm{p}}=0.5$. The value 


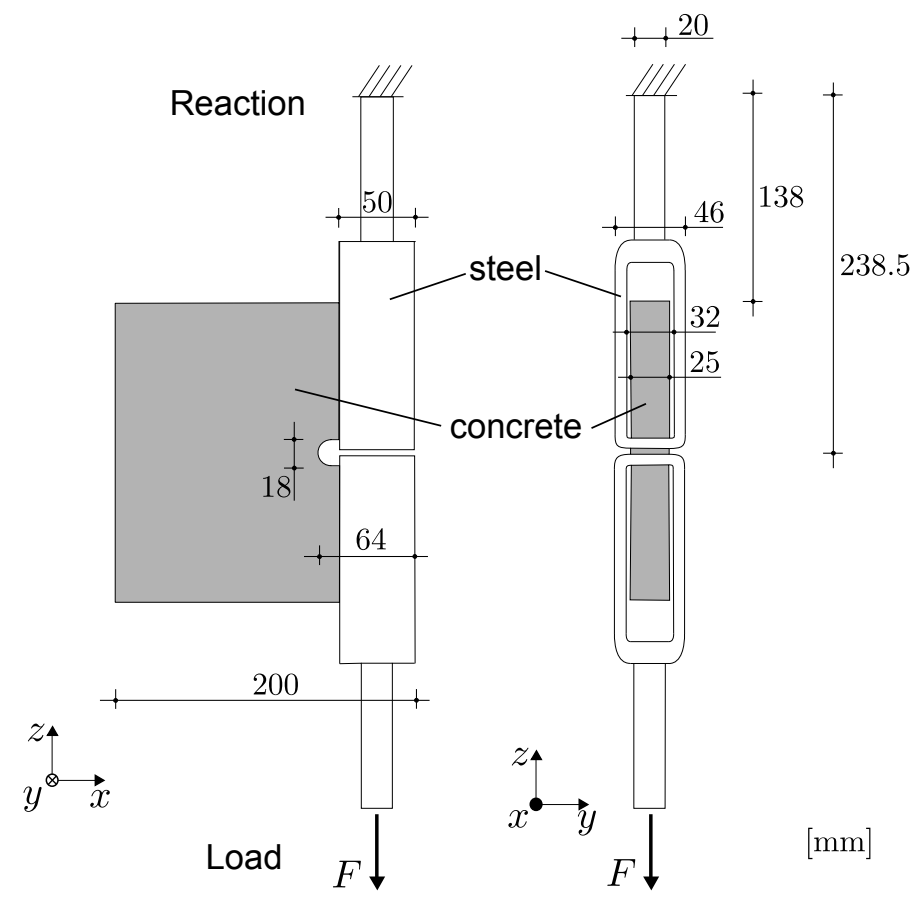

Figure 3: Geometry and loading setup of the compact tension test according to Ožbolt et al. (2013).

of the fracture energy $G_{\mathrm{F}}$ determined from the Model Code 2010 is greater than the value used in the analyses reported in Ožbolt et al. (2013).

In Figure 4, the reaction force versus time of the analyses for the four displacement rates and three meshes are shown and compared to the available experimental results for $v=1.4$ and $v=4.3 \mathrm{~m} / \mathrm{s}$. The experimental curves are shifted along the time axis, so that the times of the first peak for analyses and experiments coincide with the one of the experiments. The model provides mesh insensitive results for the first part of the analyses. There is also a reasonable agreement with the experimental results. In particular, for the highest displacement rate, the experimental results agree very well both with regard to the reaction force and the time evolution of this force. The model predicts also well the rate dependence of the maximum reaction force. For the quasi-static case $(v=0.01 \mathrm{~m} / \mathrm{s})$, the maximum reaction force is $2.52 \mathrm{kN}$. For the highest displacement rate $(v=4.3 \mathrm{~m} / \mathrm{s})$, this value increases to $5.32 \mathrm{kN}$.

In the analyses, cracks are modelled as regions of localised strains. In Figure 5, the crack patterns for the fine meshes for the four dis- placement rates are shown in the form of maximum strain contour plots. For $v=0.5,1.4$ and $4.3 \mathrm{~m} / \mathrm{s}$, the experimental crack patterns reported in Ožbolt et al. (2013) are shown as well.

The crack patterns obtained in the model are strongly influenced by the displacement rate. For the smallest displacement rate $(v=$ $0.01 \mathrm{~m} / \mathrm{s}$ ), the crack is aligned perpendicular to the loading direction as expected from a quasistatic compact tension test. As the displacement rate increases, the crack direction deviates slightly from this perpendicular direction. For $v=1.4 \mathrm{~m} / \mathrm{s}$ crack branching occurs and for $v=4.3 \mathrm{~m} / \mathrm{s}$, two cracks start at the notch tip. These numerical crack patterns are similar to those observed in experiments. However, in the experiments, crack branching only occurs for $v=4.3 \mathrm{~m} / \mathrm{s}$. It is expected that fracture energy has a strong effect on the displacement rate at which crack branching is observed. Therefore, it is planned to investigate further the influence of fracture energy on the crack patterns in these tests. 


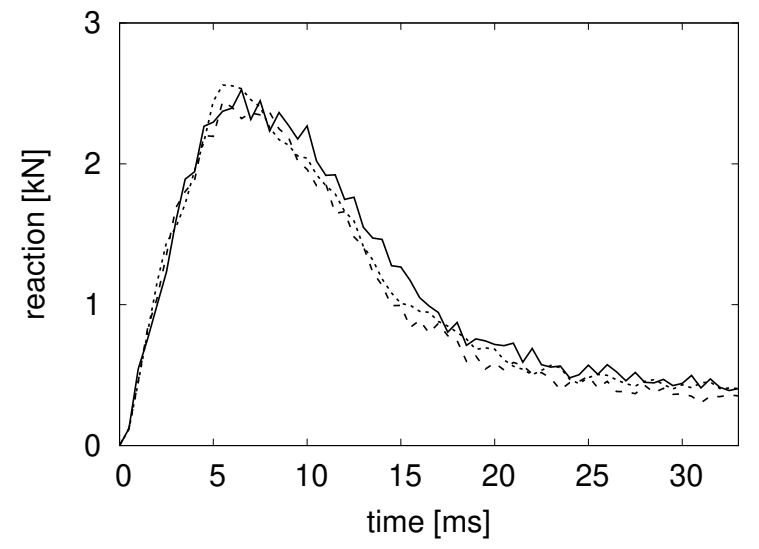

(a)

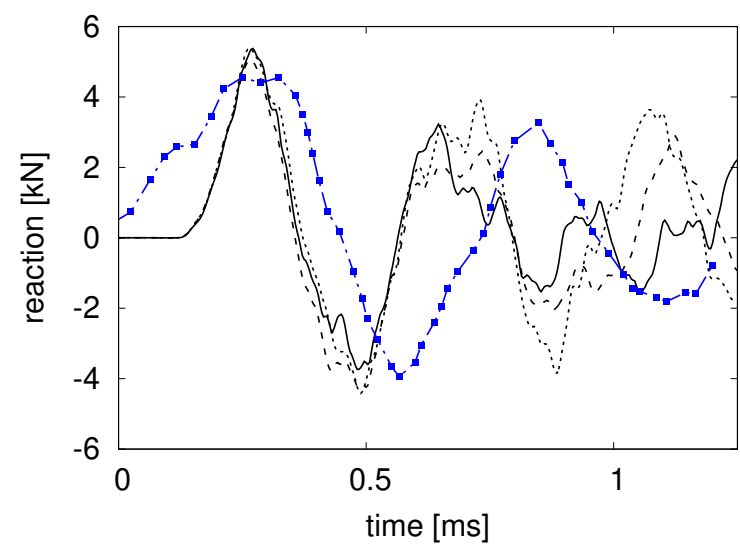

(c)

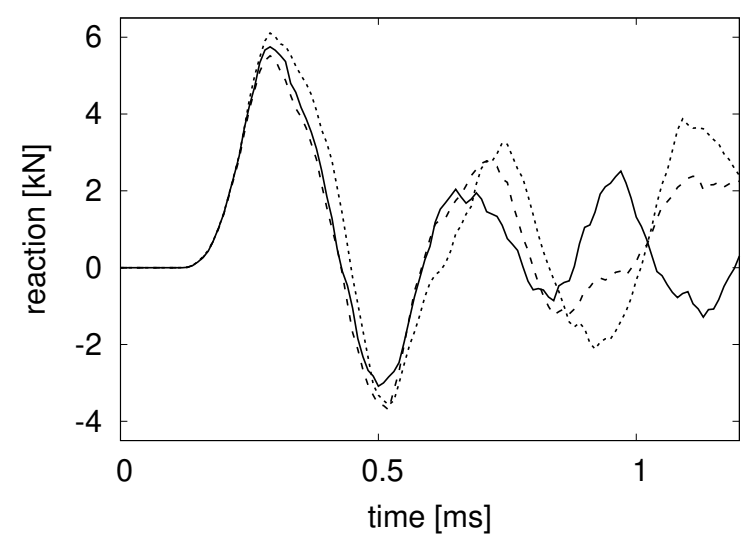

(b)

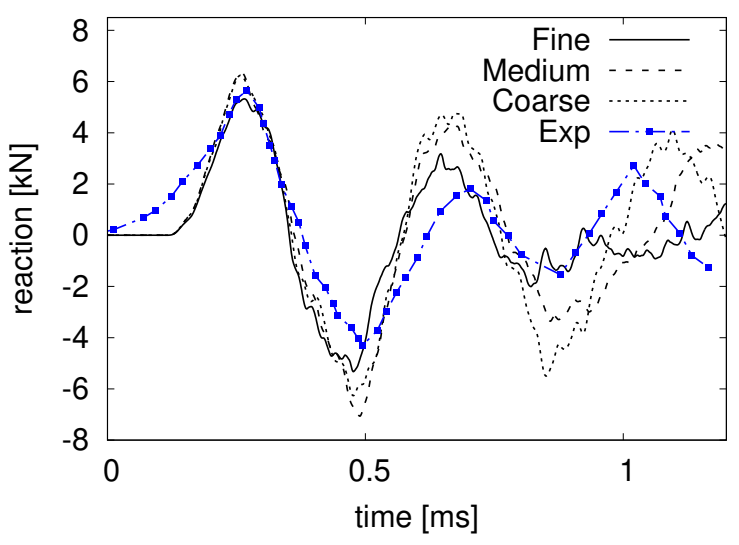

(d)

Figure 4: Reaction force versus time for the compact tension test with velocities (a) $v=0.01$, (b) $v=0.5$, (c) $v=1.4$ and (d) $4.3 \mathrm{~m} / \mathrm{s}$. The experimental results are from Ožbolt et al. (2013).

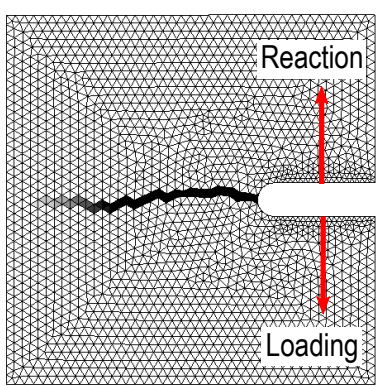

(a)

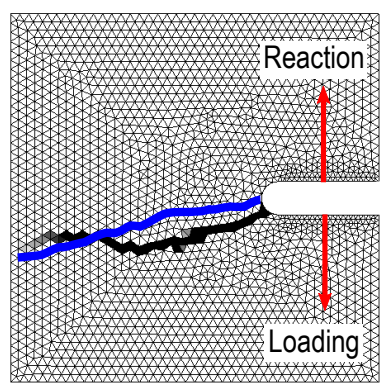

(b)

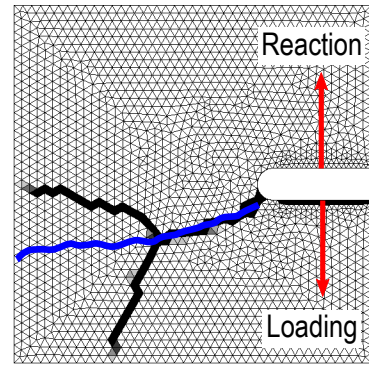

(c)

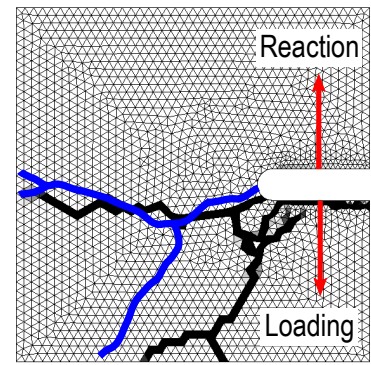

(d)

Figure 5: Crack patterns for (a) $v=0.01$, (b) $v=0.5$, (c) $v=1.4$ and (d) $4.3 \mathrm{~m} / \mathrm{s}$ for the fine mesh in the form of maximum strain contour plots. Here, black indicates a maximum principal strain which corresponds to crack openings greater than $0.1 \mathrm{~mm}$. Experimental crack patterns are shown in blue. 


\subsection{REINFORCED CONCRETE SLAB}

The second test consists of a reinforced concrete slab subjected to air blast for which the experimental results were reported in Thiagarajan and Johnson (2014). This setup was part of a blind simulation competition which was discussed in detail in Schwer (2014a) and was also analysed with the old version of CDPM2 in Ekström (2016). The slab has a two-directional reinforcement arrangement as shown in Figure 6. In the experiment, the slab was positioned vertically in a test frame, with the side which is away from the pressure resting on a support frame. This support frame is modelled in the analyses by the supporting beams shown in Figure 6. To avoid that the slab falls out of the frame during the experiment, it was kept in position by two additional support beams on the pressure side. In Thiagarajan and Johnson (2014), it was stated that gap between the additional support beams on the pressure side and the slab was so large that it could be assumed that the slab was simply supported in the experiments. Therefore, in the analysis setup in Figure 6, the support beams on the pressure side are not modelled. However, the pressure which is used to represent the air blast is not applied over the area in which these additional support beams are positioned in the experiments.

Concrete is modelled again by tetrahedral constant strain elements using the new version of CDPM2 described in section 2.1. Only one mesh size is used for these analyses with an element edge length of $10 \mathrm{~mm}$. This corresponds to the medium mesh size used for the compact tension test in section 3.1. The input parameters for CDPM2 are determined based on a uniaxial compressive strength of $f_{\mathrm{c}}=34.5 \mathrm{MPa}$ reported in Schwer (2014a). Model Code 2010 (CEB-FIP 2012) is then used to determine the other material properties as $E=32.5 \mathrm{GPa}, f_{\mathrm{t}}=$ $2.7 \mathrm{MPa}, G_{\mathrm{f}}=138 \mathrm{~J} / \mathrm{m}^{2}$. Poisson's ratio is set to $\nu=0.2$. The strain threshold for compressive softening is set to $\varepsilon_{\mathrm{fc}}=1 \times 10^{-2}$ to avoid premature compressive failure on the pressure side. The other parameters are set to their recommended default values for the strain rate de- pendent version of CDPM with $H_{\mathrm{p}}=0.5$.

For the reinforcing steel, circular crosssection frame elements are used with a diameter of $\phi=9.5 \mathrm{~mm}$. The constitutive model for the steel is the modified Johnson-Cook model described in section 2.2. The elastic input parameters are chosen as $E=200 \mathrm{GPa}$ and $\nu=0.3$. The parameters for the hardening response are set to $A=415 \mathrm{MPa}, B=550 \mathrm{MPa}, N=0.21$ to match the stress-strain curves of the steel reported in Schwer (2014a). The parameter for the rate dependence of steel is set to $C=0.017$. The supports are assumed to be linear-elastic with $E=200 \mathrm{GPa}$ and $\nu=0.3$.

The effect of the air blast is modelled by applying a time dependent pressure over the area of the slab shown in Figure 6. The evolution of the pressure with time shown in Figure 7 a was taken from Schwer (2014a).

The results are shown in form of the vertical mid-point deflection at the pressure side of the slab versus time in Figure $7 \mathrm{p}$ and crack patterns in Figure 8.

From Figure $7 \mathrm{~b}$, it can be seen that the initial response of the slab obtained in the model agrees well with the experimental results reported in Thiagarajan and Johnson (2014). However, the maximum displacement obtained with the model is less than the one recorded in the experiment.

The crack pattern in Figure 8 at a displacement marked in Figure $7 \mathrm{p}$ shows tensile cracks at the bottom of the slab, which are generated at an early state of the analysis. Then, at a later stage, shear cracks are created which are visible in the side view of the cracks pattern. A shear failure occurs on the left hand side of the slab. Although the bending cracks agree well with experimental results, this shear failure was not reported in Thiagarajan and Johnson (2014). In future studies it is intended to investigate this aspect further.

\section{CONCLUSIONS}

In this work, the damage-plasticity model CDPM2 is extended to the modelling of concrete subjected to dynamic loading and applied 

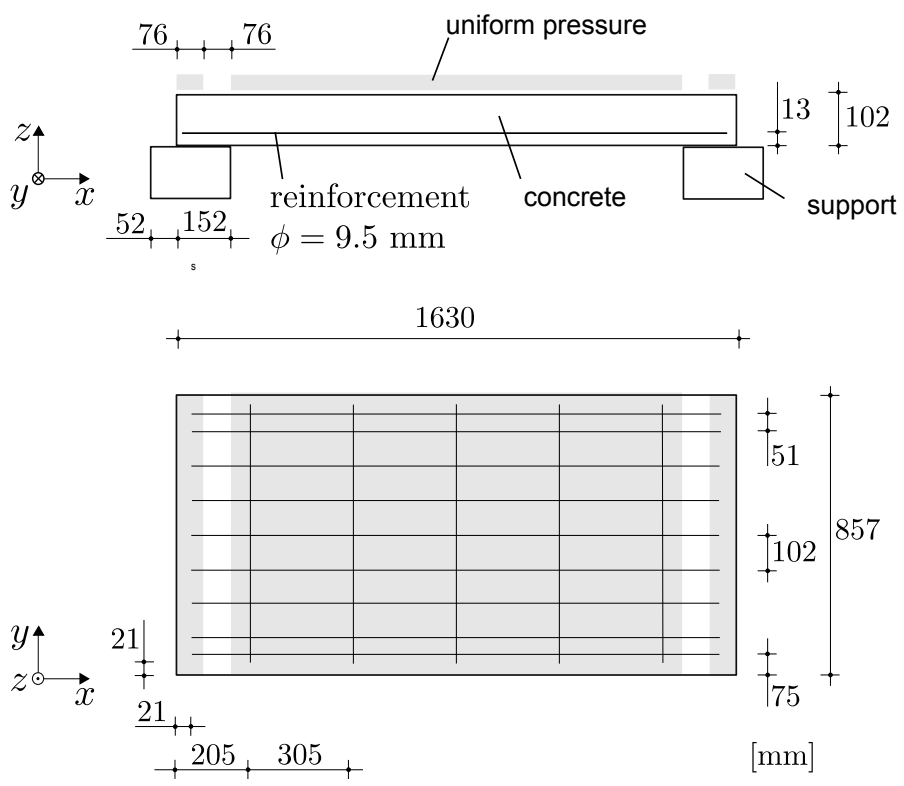

Figure 6: Geometry and loading setup of the single reinforced concrete slab subjected to blast loading according to Thiagarajan and Johnson (2014).

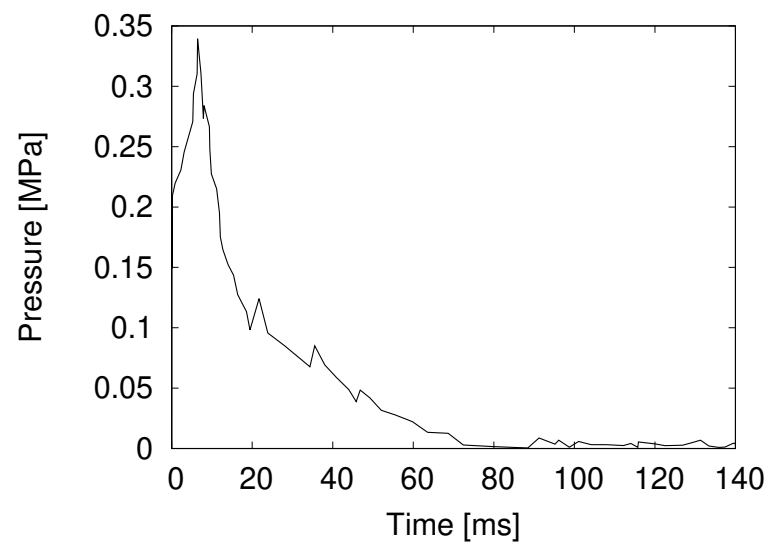

(a)

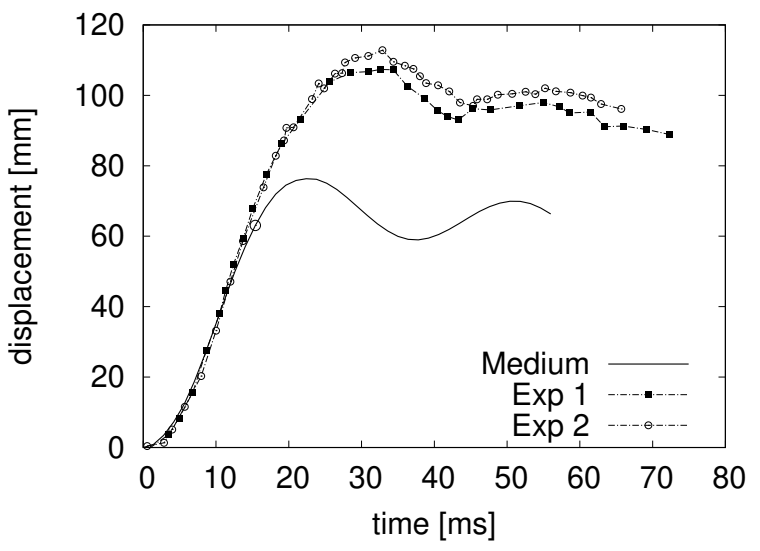

(b)

Figure 7: Reinforced concrete slab subjected to air blast: a) Pressure versus time used to model the effect of the airblast, b) model midpoint displacement versus time compared to experimental results reported in Thiagarajan and Johnson (2014). 

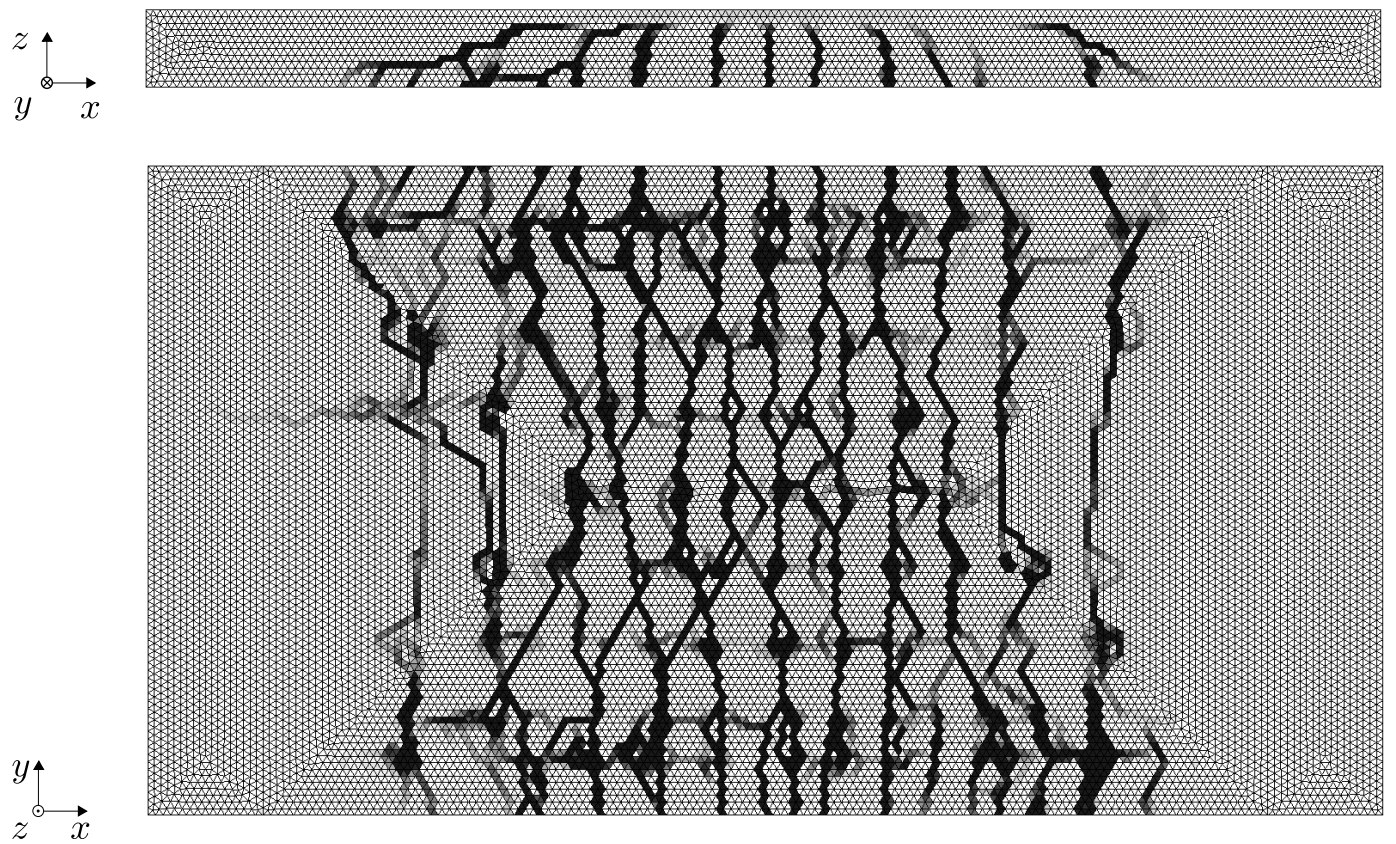

Figure 8: Reinforced concrete slab subjected to air blast: Contour plots of the maximum principal strain representing crack patterns at the side and bottom of the slab. Here, black shows a maximum principal strain which corresponds to crack openings greater than $0.5 \mathrm{~mm}$.

to the analyses of a dynamic compact tension test and a reinforced concrete slab subjected to air blast. The new version of CPDM2 represents overall well the rate dependent crack patterns observed in the experiments of the compact tension test. The reaction versus time response is independent of the mesh size for the first part of the analysis. For the reinforced concrete slab, the bending cracks and initial displacement versus time response observed in the experiments are well represented. However, the maximum deflection of the slab is underestimated. Also, the model predicts a shear failure which has not been reported in the experiments.

\section{References}

Bažant, Z. P. \& B.-H. Oh (1983). Crack band theory for fracture of concrete. Materials and Structures 16, 155-177.

CEB-FIP (2012). CEB-FIP Model Code 2010, Design Code.

Cusatis, G. (2011). Strain-rate effects on concrete behavior. International Journal of Impact Engineering 38, 162-170.

de Pedraza, V. R., F. Galvez, \& D. C. Franco (2018). Measurement of fracture energy of concrete at high strain rates. In EPJ Web of Conferences,
Volume 183, pp. 02065.

Ekström, J. (2016). Concrete structures subjected to blast loading. Licentiate thesis, Chalmers University of Technology.

Grassl, P. (2016). Quasi-static three point bending IS-Dyna analyses with MAT CDPM (MAT 273) using tetra-and hexahedral meshes. Research report, School of Engineering, University of Glasgow.

Grassl, P., M. Johansson, \& J. Leppänen (2018). On the numerical modelling of bond for the failure analysis of reinforced concrete. Engineering Fracture Mechanics 189, 13-26.

Grassl, P., U. Nyström, R. Rempling, \& K. Gylltoft (2011). A damage-plasticity model for the dynamic failure of concrete. In 8th International Conference on Structural Dynamics, Leuven, Belgium.

Grassl, P., D. Xenos, U. Nyström, R. Rempling, \& K. Gylltoft (2013). CDPM2: A damageplasticity approach to modelling the failure of concrete. International Journal of Solids and Structures 50(24), 3805-3816.

Jirásek, M. \& M. Bauer (2012). Numerical aspects of the crack band approach. Computers and Structures 110-111, 60-78.

Jirásek, M. \& Z. P. Bažant (2002). Inelastic Analysis of Structures. Chichester: John Wiley and Sons. 
Johnson, G. R. \& W. H. Cook (1985). Fracture characteristics of three metals subjected to various strains, strain rates, temperatures and pressures. Engineering fracture mechanics 21(1), 31-48.

Menétrey, P. \& K. J. Willam (1995). A triaxial failure criterion for concrete and its generalization. ACI Structural Journal 92, 311-318.

Ožbolt, J., A. Sharma, B. İrhan, \& E. Sola (2014). Tensile behavior of concrete under high loading rates. International Journal of Impact Engineering 69(12), 55-68.

Ožbolt, J., J. Bošnjak, \& E. Sola (2013). Dynamic fracture of concrete compact tension specimen: Experimental and numerical study. International Journal of Solids and Structures 50(2526), 4270-4278.

Pereira, L. F., J. Weerheijm, \& L. J. Sluys (2017). A numerical study on crack branching in quasibrittle materials with a new effective ratedependent nonlocal damage model. Engineering Fracture Mechanics 182, 689-707.

Pietruszczak, S. T. \& Z. Mróz (1981). Finite element analysis of deformation of strain-softening materials. International Journal for Numerical Meth- ods in Engineering 17(3), 327-334.

Schwer, L. (2014a). Blind blast simulation: Prediction of air blast loaded concrete slab response using six ls-dyna concrete models. In 13th International LS-DYNA Conference, pp. 1-32.

Schwer, L. (2014b). Modeling rebar: The forgotten sister in reinforced concrete modelling. In 13th International LS-DYNA Conference, pp. 128. Dynamore.

Thiagarajan, G. \& C. F. Johnson (2014). Experimental behavior of reinforced concrete slabs subjected to shock loading. ACI Structural Journal 111(6), 1407-1417.

Willam, K., N. Bićanić, \& S. Sture (1986). Composite fracture model for strain-softening and localised failure of concrete. In E. Hinton and D. R. J. Owen (Eds.), Computational Modelling of Reinforced Concrete Structures, Swansea, pp. 122-153. Pineridge Press.

Xenos, D. \& P. Grassl (2016). Modelling the failure of reinforced concrete with nonlocal and crack band approaches using the damage-plasticity model CDPM2. Finite Elements in Analysis and Design 117, 11-20. 